The number of organs donated by older people doubled and waiting time for elderly recipients decreased following introduction of the ESP, supporting the overall effectiveness of such a system.

Original article Frei U et al. (2008) Prospective agematching in elderly kidney transplant recipients-a 5-year analysis of the Eurotransplant Senior Program. Am J Transplant 8: 50-57

\section{Transcriptome analysis is a superior method for predicting delayed kidney graft function}

Prediction of the quality of organs donated for transplantation might soon be improved by microarray analysis of the genes expressed in biopsy samples. Mueller et al. evaluated the potential of 'transcriptomics' to determine the risk of delayed renal graft function (DGF), by comparing the results of automated RNA analyses with those from clinical and histopathological examinations. Tests were performed on 87 post-reperfusion biopsy samples taken from kidneys from 31 deceased donors and 45 living donors.

Categorization of organs on the basis of differential expression of genes (from a total pool of almost 55,000 on each microarray 'chip') yielded three distinct groups: one comprising kidneys from living donors; and two comprising organs from deceased donors (DD1 and DD2). Onset of function of allografts from the DD2 group was more likely to be delayed than that of kidneys from the DD1 and living-donor groups $(P<0.05$ and $P<0.001$, respectively). DD2 samples had increased expression of genes associated with complement, chemokines, collagen, inflammation, and antigen processing, but fewer transcripts involved in metabolism and transport.

Standard clinical and histopathological assessments detected no difference in the likelihood of DGF among the 31 deceased donors. By contrast, a transcriptome-based score reflected the susceptibility for DGF and revealed a continuum from best-functioning to poorest-functioning kidneys. The authors conclude, therefore, that microarray analysis of the transcriptome is a better indicator of organ quality and DGF risk than standard examination and could identify potentially useful kidneys that might otherwise be discarded.

Original article Mueller TF et al. (2008) The transcriptome of the implant biopsy identifies donor kidneys at increased risk of delayed graft function. Am J Transplant 8: 78-85

\section{Peritubular capillary C4d deposition not linked with graft outcome in late acute rejection}

Diffuse peritubular capillary (PTC) deposition of complement degradation component C4d is a marker of acute rejection in the early post-transplantation period and is associated with poor renal graft survival. Satoskar and colleagues have reported that diffuse PTC C4d deposition might not, however, be predictive of poor graft outcome in late acute rejection episodes (i.e. those occurring $\geq 1$ year after transplantation).

This retrospective study included biopsy samples from 40 renal allograft recipients who experienced a first episode of acute rejection $\geq 1$ year after undergoing transplantation. Twenty C4d-positive biopsy samples (>80\% staining) and $20 \mathrm{C} 4 \mathrm{~d}$-negative biopsy samples ( $<40 \%$ staining) were included, with each group matched for age, sex, maintenance and post-rejection immunosuppression, baseline serum creatinine levels (before rejection was diagnosed), length of time between transplantation and diagnostic biopsy, and chronic allograft damage index in the biopsy sample.

A mean of 20 months of follow-up data were available for each patient. Graft survival was similar in the C4d-positive and C4d-negative groups $(P=0.565)$. Among the 21 patients with functioning grafts at the end of the study period, serum creatinine levels were similar in the two groups, as were increases in serum creatinine levels between baseline and the end of follow-up. The degree of PTC margination of inflammatory cells and the incidence of transplant glomerulopathy were both significantly higher in the C4d-positive group than in the C4d-negative group $(P=0.004$ and $P=0.020$, respectively). The degree of plasma cell infiltration was similar in the two groups.

Original article Satoskar AA et al. (2007) Peritubular capillary C4d staining in late acute renal allograft rejectionis it relevant? Clin Transplant 22: 61-67 